\title{
PREMIÈRES OBSERVATIONS
}

\section{SUR LE COMPORTEMENT ALIMENTAIRE DES PORCS}

\author{
PAR

\section{R. FEVRIER} \\ Station de Recherches sur l'Élevage, \\ Centre national de recherches zootechniques, Jouy-en-Josas
}

Nous avons précédemment rappelé (8) que la vitesse de croissance et l'utilisation de la nourriture étaient étroitement liées au volume de l'ingestion. Celui-ci dépend à son tour de l'appétit de l'animal, qui est fonction d'une certaine "aptitude " individuelle, mais aussi de nombreux facteurs, dont plusieurs auteurs se sont efforcés de préciser l'importance. Citons pour mémoire : l'état de l'animal : croissance, état adulte, gestation (COLE et Har'T (5)), le niveau d'alimentation (KATZ, (II)), la nature du régime antérieur (HARRIS et al, (IO) ; Tribe et Gordon (I5) ; Young (I6)) et l'effet collectif (Harlow (9)). Mais l'un des plus importants demeure la nature de la ration offerte, qui peut exciter de façon extrêmement variable l'appétit de l'animal. Cette propriété, liée à l'aliment, est habituellement désignée sous le nom de sapidité, appétence, et à l'image des Anglo-Saxons, palatibilité.

Il serait extrêmement important de pouvoir caractériser l'appétence d'un aliment, d'une façon relative ou d'une façon absolue. En effet, l'étude comparative de divers aliments nous apporterait des renseignements précieux sur la psycho-physiologie de l'appétit, et sa connaissance nous permettrait de composer des rations mieux adaptées au but recherché, qu'il soit d'ordre pratique ou d'ordre expérimental. Or, il est maintenant bien acquis que la palatibilité n'est pas le témoin infaillible de la valeur de l'aliment pour l'animal (AschkeNASY-LELU (I)).

Les zootechniciens accordent une importance considérable à cette qualité, tels Carroli et Krider (3) et David son (6). Brody (2) arfirme: " Palatibility is an agriculturally important characteristic because the greater palatibility of a food, the greater its consumption, the more rapid the productive process, the less overhead maintenance cost and the higher the economy per unit product ".

Or, la mesure de l'appétence d'une ration soulève de nombreuses diffcultés, et les tentatives effectuées dans ce sens ne sont pas très encourageantes. CokAyne (4) cite le travail de cinq chercheurs sur la palatibilité de l'Agropyrum Scabrum, qui donnent une note variant de I à 5. FAGAN et JoNE (7) établissent 
un classement différent de celui de MiLTon (I4), avec des animaux de même type et les mêmes espèces botaniques de plantes qui, notons-le, peuvent présenter une sapidité très différente selon les conditions dans lesquelles elles se sont développées ; TrIBE et GoRDON (I5) concluent qu'il est erroné d'attribuer à une plante donnée une valeur fixe de palatibilité. On peut néanmoins se demander si, dans des conditions normales de vie, la hiérarchie des palatibilités des différents aliments ne se conserve pas. Dans la négative, on peut étudier les facteurs qui peuvent la modifier. Il suffirait alors de définir les conditions dans lesquelles est effectuée cette mesure, pour qu'elle puisse être reproduite. La connaissance d'un tel classement, malgré ses imperfections, rendrait de nombretux services.

C'est dans ce but que nous avons élaboré un programme de recherches, dont la réalisation devra attendre l'achèvement des installations en cours à Jouy-en-Josas. Nous avons néanmoins procédé à quelques observations préalables à la Station de Bois-Corbon ; c'est l'une d'elles que nous relatons ici.

La première idée que l'on peut avoir pour classer des aliments selon leur palatibilité, est de comparer les quantités de différents aliments consommés par le même animal et de les rapporter à la quantité d'aliment témoin consommée. Nous avons utilisé cette méthode, malgré les réserves que l'on a pu faire sur sa valeur à la suite des travaux de MILLER, BAILEY et Stevenson (I3) qui, à propos de rats ayant subi des lésions hypothalamiques, parlent "d'une faim sans appétit".

La consommation varie-t-elle dans le même sens pour tous les animaux, quand on passe de l'aliment témoin à l'aliment expérimental ? L'ampleur de cette variation est-elle constante? Dépend-elle de l'âge de l'animal ?

Telles étaient les questions auxquelles notre étude préliminaire devait apporter des éléments de réponse.

\section{Matériel et méthodes}

Nous disposions de 8 groupes de porcs Large White Yorkshire, pesant de 40 à $85 \mathrm{~kg}$ environ. Chaque groupe comprenait 3,4 ou 5 animaux de poids voisins.

Ils étaient habitués à l'aliment standard que nous avons décrit ailleurs (I2). Nous avons confectionné un autre mélange alimentaire (aliment $B$ ), présentant des caractéristiques analytiques également conformes aux normes de l'alimentation rationnelle, mais à partir de matières premières différentes. Le tableau I donne les résultats de l'analyse effectuée sur ces deux mélanges :

TABLEAU I

Matière sèche $\ldots \ldots \ldots \ldots \ldots \ldots \ldots \ldots$

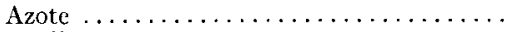

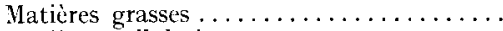

Matières cellulosiques ...............

Matières minérales

Aliment standard

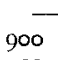

900
29,3

34

65

74

Aliment 13 .

898

$3^{2}, 5$

62

92

82 
Notre objectif était d'étudier le comportement alimentaire des animaux lors de la substitution de l'aliment B à l'aliment standard. La consommation de l'animal augmentant au fur et à mesure qu'il prend de l'âge, nous avons comparé la consommation d'aliment $B$ pendant une période de 4 jours (période III) à la moyenne de consommation de l'aliment standard pendant deux périodes de 4 jours également, situées l'une (période I) avant et l'autre (période V) après la période III.

A chaque changement de régime, une période de 3 ou 4 jours (périodes II et IV) dite " période d'adaptation " était laissée à l'animal pour lui permettre de s'accoutumer à sa nouvelle ration.

Le tableau II représente le plan d'alimentation que nous venons de décrire :

TABLEAU II

$\begin{array}{rrcl}\text { Période } & \text { Durée en jours } & \text { Aliment distribué } & \text { Observations } \\ - & - & - & \text { Standard } \\ \text { II } & 4 & \text { B } & \text { Mesure de la consom. } \\ \text { II } & 4 & \text { Adaptation } \\ \text { III } & 4 & \text { B } & \text { Mesure de la consom. } \\ \text { IV } & 3 & \text { Standard } & \text { Adaptation } \\ \text { V } & 4 & \text { Mesure de la consom. }\end{array}$

Ces mélanges alimentaires, additionnés de 3 fois environ leur poids d'eau, étaient distribués en trois repas, et la quantité allouée était fixée en fonction de ce que l'animal pouvait consommer en 20 minutes. Une observation attentive de l'appétit des animaux permettait d'éviter les refus, qui constituèrent l'exception : au cours de cette étude qui comporta la distribution de 288 rations, 7 cas de refus seulement furent enregistrés. Dans ce cas, les quantité non ingérées étaient recueillies, la quantité de matière sèche était calculée après passage à l'étuve et la correction était effectuée pour déterminer, à partir de la ration distribuée, l'exacte quantité de nourriture ingérée par les animaux.

\section{Résultats obtenus}

Le tableau III donne les résultats obtenus au cours de cette étude :

TABLEAU III

\begin{tabular}{|c|c|c|c|c|c|c|c|}
\hline \multirow[b]{2}{*}{$\begin{array}{l}\text { No du } \\
\text { groupe }\end{array}$} & \multirow{2}{*}{$\begin{array}{c}\text { Nombre } \\
\text { de } \\
\text { porcs }\end{array}$} & \multicolumn{3}{|c|}{$\begin{array}{c}\text { Consommation moyenne } \\
\text { quotidienne par animal }(\mathrm{kg})\end{array}$} & \multicolumn{3}{|c|}{$\begin{array}{c}\text { Durée moyenne des } \\
\text { repas (minutes) } \\
\text { Périodes }\end{array}$} \\
\hline & & $\begin{array}{l}\text { Période I } \\
\text { (stand.) }\end{array}$ & $\begin{array}{l}\text { Période III } \\
\text { (B) }\end{array}$ & $\begin{array}{l}\text { Période V } \\
\text { (stand.) }\end{array}$ & I & III & V \\
\hline- & - & - & - & 一 & - & - & - \\
\hline I & 4 & 3,15 & $2,5^{6}$ & $3,4 \mathrm{I}$ & 13 & I I & I6 \\
\hline 2 & 4 & 3,66 & 2,69 & 3,53 & I 2 & 10 & I3 \\
\hline 5 & 4 & I, 88 & I, 44 & $2, I 3$ & I3 & I 2 & I I \\
\hline 7 & 4 & 2,63 & 2,22 & 2,75 & 12 & I 5 & Io \\
\hline I I & 5 & 3,80 & $2,5^{2}$ & $4, \infty$ & I 2 & I3 & I I \\
\hline I3 & 4 & 2,03 & $\mathbf{I}, 69$ & 2,28 & I I & I 2 & I0 \\
\hline I 4 & 4 & 2,63 & $2,3 \mathrm{I}$ & $3, \infty$ & 9 & 8 & 8 \\
\hline I 5 & 3 & 3,24 & $2,4 \mathrm{I}$ & $3, \infty$ & I 8 & 16 & 13 \\
\hline par j & & 2,89 & 2,23 & 3,04 & 12,3 & 12,0 & I I, \\
\hline
\end{tabular}

D'une façon constante, l'aliment $\mathrm{B}$ a été moins bien accepté que l'aliment standard. 
Le tableau IV permet de comparer la consommation de l'aliment $B$ à la consommation de l'aliment standard. En effet, on peut admettre que, pour ce court intervalle de temps, la consommation de nourriture par animal s'accroit selon une loi linéaire, toutes choses égales par ailleurs. On peut donc supposer que la moyenne des consommations d'aliments des périodes I et $\mathrm{V}$ représente la consommation d'aliment que l'on aurait observée dans la période III si le régime n'avait pas été modifié (graphique I).

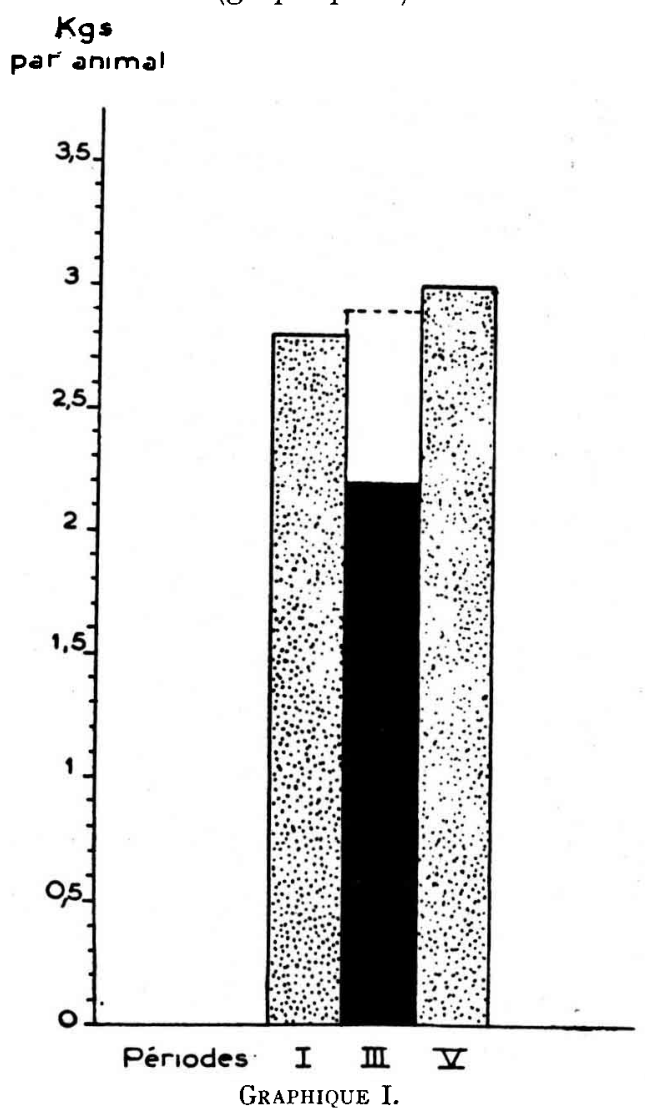

Consommation moyenne par animal au cours dee 3 périodes expérimentales

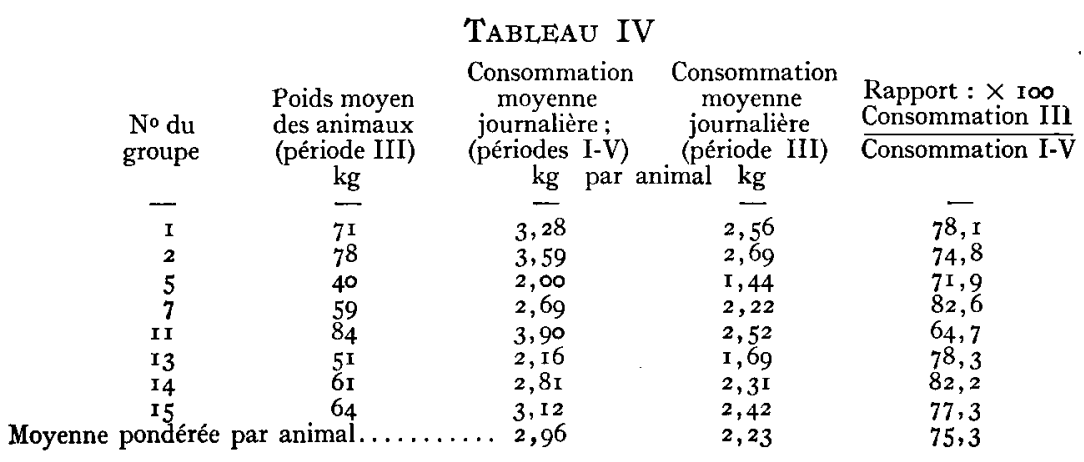





les animaux d'un poids moyen $(60 \mathrm{~kg})$ qui ont réagi le moins nettement au changement de nourriture. En portant sur un graphique. en abscisses les poids moyens des animaux du groupe et en ordonnées les valeurs correspondant au rapport :

$$
\frac{\mathrm{C} \cdot \mathrm{III}}{\mathrm{C} \cdot \mathrm{I}-\mathrm{V}}
$$

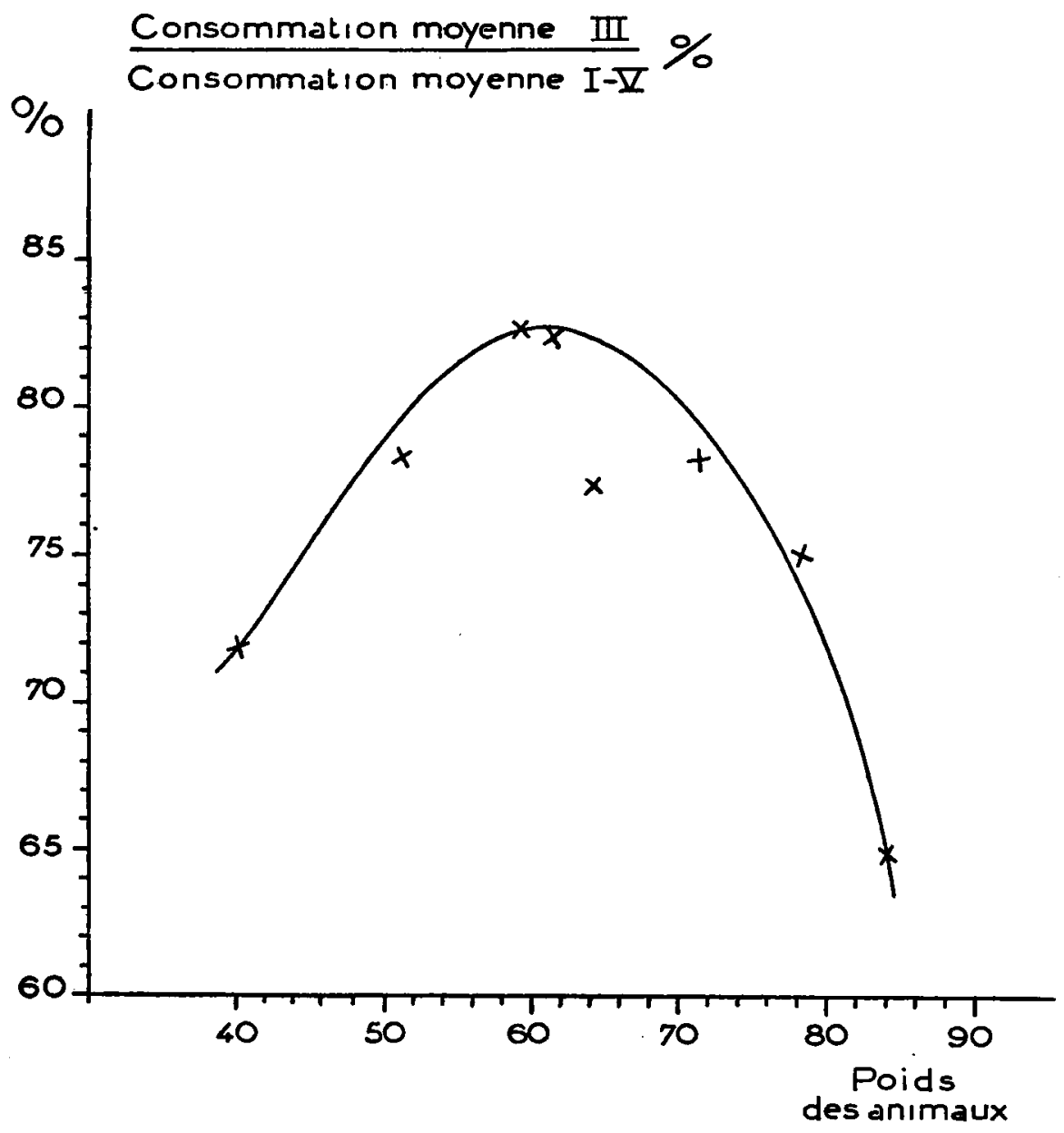

GRAPHIQUE II.

nous avons obtenu une courbe (graphique II), qui s'élève régulièrement jusqu'aux ordonnées correspondant aux animaux de $60 \mathrm{~kg}$ environ, puis $\mathrm{qu}^{\mathrm{i}}$ diminue ensuite avec la même régularité. Il semble donc que les animaux de ce poids sont les moins troublés dans leur appétit par les changements de régime, tandis que les sujets plus légers ou plus lourds réagissent par une 
réduction de consommation, d'autant plus forte que leur poids est plus éloigné de l'optimum. Le point aberrant du graphique correspond au groupe I5 $_{5}$ dont, précisément, la consommation a diminué de la période $\mathrm{I}$ à la période $\mathrm{V}$ et pour lequel notre hypothèse de calcul n'est pas vérifiée. On peut donc penser que, si son appétit s'était comporté de façon " normale ", le point représentatif se serait probablement placé sur la courbe ou dans son voisinage immédiat.

Bien que, ce cas mis à part, les points se placent sur une courbe à peu près régulière, le nombre limité de groupes, l'écart relativement réduit entre les poids moyens (40-84 kg) et l'absence d'alimentation individuelle des sujets, ne permettent pas de considérer cette conclusion comme acquise, et les travaux que nous espérons entreprendre prochainement nous permettront de vérifier la valeur de cette hypothèse.

\section{Résumé}

I. - En substituant, dans la ration de 8 groupes de porcs de 40 à $85 \mathrm{~kg}$ un aliment $B$ à un autre, auquel ils étaient habitués, nous avons observé une diminution générale de la consommation.

2. - En comparant, pour chaque groupe d'animaux, la consommation de l'aliment $\mathrm{B}$ pendant une période de 4 jours à la consommation de l'aliment standard avant et après cette période, nous avons obtenu un rapport dont la valeur varie de 64,7 à 82,6 p. Ioo et dont la moyenne pondérée est de 75,3 .

3. - Il semble y avoir une relation entre la valeur de ce rapport et le poids de l'animal : elle est maxima pour un poids voisin de $60 \mathrm{~kg}$ et s'abaisse régulièrement pour les poids inférieurs et supérieurs.

4. - Ces résultats devront être confirmés par une étude portant sur un plus grand nombre de sujets alimentés individuellement et présentant une gamme de poids plus large encore.

\section{BIBLIOGRAPHIE}

(I) Aschkenasy-Lelu (P.). - - L'ingestion alimentaire en fonction de la composition du régime. Application à la régulation du besoin azoté chez le rat. Ann. Nutrit. Alim. 4-5, 453, I 95 I.

(2) BRODY (S.). - Bioenergetics and growth (Reinhold Publ. Corpor. N. Y.), 1945.

(3) Carroll (W. E.) et Krider (J. L.). - Swine production. (McGraw-Hill Book Co, N. Y.), I95०.

(4) Cokayne (L.). - N.Z.J. Agric., 18, 32 I, 1919. in Milton (W. E. J.). - Emp. J. Exp. Agric. 2. 51 1 19.34 .

(5) Cole (H. H.) et HaRT (G. H.). - The effect of pregnancy and lactation on growth in the rat. Amer. J. Physiol, 123, $589,1938$.

(6) Davidson (H. R.). - The production and marketing of pigs. (Longmans, Green et $\mathrm{C}^{\circ}$ ), 1946.

(7) Fagan (T.W.) et Jones (M. G.). - Welsh pl. breeding Stat. Bul. H. 3.ig24. In Milton (14)

(8) FÉVRIER (R.). - L'indice de consommation est-il le témoin fidèle de l'efficacité d'une ration? Ann. Zootechnie, I-I75- I952.

(9) Harlow (H. F.). - Social facilitation of feeding in the albino rat. J. Genetic Psychol., 41, 2 I I, 1932 . 
(io) Harris (L. J.), Clay (J.), Hargreaves (F. J.) et Ward (A.). - Appetite and choice of diet. The ability of the Vitamin $B$ deficient rat to discriminate between diets containing and lacking the Vitamin. Proc. Royal Soc. London, 113 B, г6r, r933.

( II) KATZ. - Animal and Man: studies in comparative psychology. (Longmanns et Green. London)

(12) Leroy (A. M.) et FÉVRIER (R.). - Étude des aptitudes économiques et de leur transmission chez les différentes souches et races porcines. Ann. Agron., 6, I949.

(i 3) Miller (N. E.), Baldey (C. J.) et Stevenson (J. A. F.). - Decreased " hunger " but increased food intake resulting from hypothalemic lesions. Science, 112, 256- 1950.

(r4) Milton (W. E. J.). - The relative palatibility of seeds-mixtures, and a study of the influence of fertilizers on natural hill pastures. Emp. J. Exp. Agric., 2, 51- I934.

(I5) Tribe (D. E.) et Gordon (J. G.). - An experimental study of palatibility. Agric. Progress, 25, I I , 94, 1950.

(I6) Young et Chaplin. - Compar. Psychol. Monograph. 18. r, 45, 1945. in AschkehasyLelu (I) 